\title{
The Implementation of the STAR Data Acquisition System using a Myrinet Network
}

\author{
J. M. Landgraf ${ }^{1}$, C. Adler ${ }^{2}$, M. J. LeVine ${ }^{1}$, A..Ljubicic, Jr. ${ }^{1}$, \\ J. M. Nelson ${ }^{3}$, M. W. Schulz ${ }^{1}$, and J. S. Lange ${ }^{2}$ \\ ${ }^{1}$ Brookhaven National Laboratory, Upton, NY 11973, USA \\ ${ }^{2}$ University of Frankfurt, August-Euler-Straße 6, D-60486 Frankfurt, Germany \\ ${ }^{3}$ University of Birmingham, Birmingham B15 2TT, United Kingdom
}

\begin{abstract}
We will present results from the first year of operation of the STAR DAQ system using a Myrinet Network. STAR is one of four experiments to have been commissioned at the Relativistic Heavy Ion Collider (RHIC) at BNL during 1999 and 2000. The DAQ system is fully integrated with a Level 3 Trigger. The combined system currently consists of 33 Myrinet Nodes which run in a mixed environment of MVME proccssors running VxWorks, DEC Alpha workstations running Linux, and SUN Solaris machines. The network will eventually contain up to 150 nodes for the expected final size of the L3 processor farm. Myrinet is a switched, high speed, low latency network produced by Myricom and available for PCI and PMC on a wide variety of platforms. The STAR DAQ system uses the Myrinet network for messaging, L3 processing, and event building. After the events are built, they are sent via Gigabit Ethernet to the RHIC computing facility and stored to tape using HPSS. The combined DAQ/L3 system processes $160 \mathrm{MB}$ events at $100 \mathrm{~Hz}$, compresses each event to $-20 \mathrm{MB}$, and performs tracking on the events to implement a physics-based filter to reduce the data storage rate to $20 \mathrm{MB} / \mathrm{sec}$.
\end{abstract}

\section{INTRODUCTION}

STAR [1] is a large detector at the Relativistic Heavy Ion Collider (RHIC) located at Brookhaven National Laboratory. During the summer of 2000 (Year 1) RHIC obtained the first Au-Au collisions at $130 \mathrm{GcV}$. The STAR DAQ $[2,3]$ can be described as a set of detector specific, custom VME cratebased readout units along with workstation based Level 3 Trigger (L3) and event building nodes connected by a highspeed network.

The readout units can be classified according to their data readout size. For detectors with large data volumes (TPC, SVT, FTPC) the readout is divided among many VME crates. Each crate contains multiple Receiver Boards [4], each of which receives data in parallel on a separate fiber. The Receiver Boards perform front end processing on the incoming data including 10-8 bit conversion, pedestal subtraction, data formatting, and cluster finding. This processing is accomplished using 3 mezzanine cards mounted on each Receiver Board, each of which contains 6 custom ASICs [5] and an $1960 \mathrm{CPU}$. In addition to the Receiver Boards, each VME crate contains one Motorola MVME 2306 Detector Broker. (DET) which contains the physical interface to the event building network. The Detector Broker controls the i960s, collects the data from the receiver boards, and presents a unified, detector-independent interface to the network. Detectors with smaller data volumes are read out directly by a
Detector Broker. These readout units reduce the incoming event size from $\sim 160 \mathrm{MB}$ to $\sim 8.5 \mathrm{MB}$. In the first year of operation of STAR there were a total of 14 Detector Brokers. The Detector Brokers can present events to the DAQ event building network at rates up to $100 \mathrm{~Hz}$.

An interesting feature of STAR is the Level 3 (L3) trigger system [6]. The L3 is a software trigger that performs tracking in real time and provides a physics based filter to reduce the output data rate to $-20 \mathrm{Mb} / \mathrm{sec}$. The tracking is performed in parallel by Sector Level 3 nodes (SL3) each of which performs tracking for a sector of the TPC. A Global Level 3 processor (GL3) performs track merging and provides the event decision. The L3 system is fully integrated with the DAQ network, receiving data from the Detector Brokers and returning an event decision to DAQ for each event. The L3 trigger is implemented on a farm of Compaq Alpha workstations running Linux. The year 1 configuration of the L 3 farm contained 12 SL3 nodes to be expanded to at least 36 nodes for year 2 .

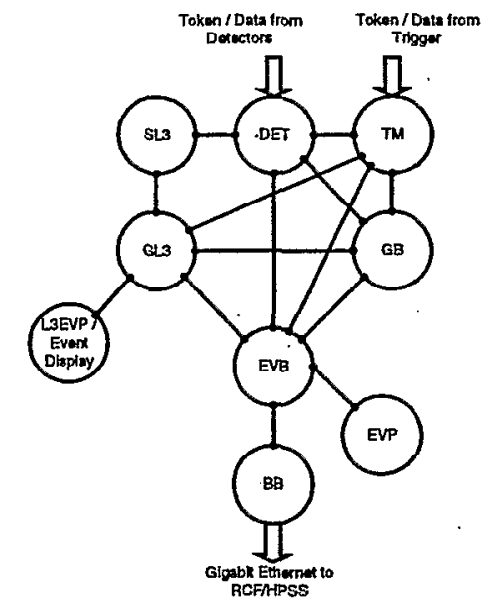

Figure 1: The functional layout of the STAR DAQ event building network. The SL3 and DET contributions represent multiple nodes as described in the text. The network is a fully connected tree. The lines represent actual message and data paths.

The functional layout of the STAR DAQ event building network is shown in Figure 1. The STAR DAQ/L3 system pipelines the data processing by allowing multiple events to be processed independently. Trigger sends a token for each event and trigger data to $D A Q$, which returns tokens after event building. These data are transferred from the Trigger DAQ Interface to the Token Manager (TM) via the VME bus. The Global Broker (GB), synchronizes events within the DAQ system and routes events accepted by the $L 3$ trigger to the Event Builder (EVB). The Event Builder gathers data banks from each DET, track data from the GL3, 
and trigger data from the Global Broker and assembles the information into events. These nodes are all implemented on MVME processors running VxWorks.

The Event Builder ships the data to the Buffer Box (BB), a Solaris workstation, which is responsible for writing the data over a Gigabit Ethernet fiber to the RHIC computing facility (RCF) where the data are stored using an HPSS Tape System. The Buffer Box also contains enough disk space to buffer $\sim 2$ hours of data in the event that the HPSS system is unavailable for short periods of time.

Finally, there are two nodes dedicated to quality assurance and online analysis. The first, the L3 Event Pool/Event Display (L3EVP) renders tracked events in real time, and can produce histogram information from the full set of input data, including events that are rejected from taping. The second is the online Event Pool (EVP), which receives full local copies of sampled of events.

In the original design of the STAR DAQ, and in the Year 0 commissioning done in 1999 , message and data transfers were performed by an SCI network. The core DAQ nodes were arranged in a loop topology, and the L3 nodes were to be arranged in separate loops connected to the DAQ network using SCI switches. Numerous concerns with the SCI network including cost, poor hardware reliability, the lack of interoperability between PCI and PMC versions of the SCI network cards, and the difficulty of debugging single-point faults in the loop topology prompted us to investigate other networks. In this paper we describe the implementation of a Myrinet [7] network in the STAR DAQ, including the STAR myriLib library. We will also discuss the porting of the EVB to Solaris workstation, a year 2 upgrade to STAR DAQ made possible by the adoption of the Myrinet network. In addition we discuss the performance and reliability of the STAR DAQ and its Myrinet network during the year $1 \mathrm{RHIC}$ run.

\section{THE MYRINET NETWORK}

Myrinet is a low-cost, high-performance, low-latency commercial network built by Myricom [8] and typically used in cluster computing applications. In this paper we discuss results using the second generation Myrinet which consists of PCI/PMC network cards connected to switches via 1.28 Gbit/sec full duplex links. A third generation network is now available as well, offering full duplex $2 \mathrm{Gbit} / \mathrm{sec}$ links.

The Myrinet network cards contain a LANai CPU that manages the transfers between the host PCI bus and the network fabric. On board SRAM is used to buffer incoming and outgoing traffic that is moved to the host and to the network by DMA engines. The LANai processor runs a Myrinet Control Program (MCP) that processes the incoming and outgoing packets. In addition, a Myrinet driver, GM, is required on the host side. A very attractive feature of Myrinet is the extent to which the network interface is implemented in software and that the source code for the MCP and the GM driver, as well as the LANai processor compilers are available from Myricom. This gives the user almost full control of the network, reduces their dependence on the manufacture, and has even led to research that would be much more difficult or impossible with other networks [9].
Myrinet switches employ cut-through wormhole routing. The route information is contained in a variable length header of port offsets that are stripped off at each switch node until the packet arrives at its destination. Flow control is accomplished with stop/go tokens inserted into the opposite flowing data link and the transit time is accommodated using a small buffer at each switch node. Because the routing information is part of the packet, the sending node needs to know the full topology of the network. This is typically arranged by designating one node as the mapper. This node periodically sends out exploratory packets and delivers the information to all nodes. While this structure allows dynamic reconfiguration of the network map if some switch becomes unavailable, it is inconvenient in our system because the unavailability of the mapper node means that the network can not be configured. For this reason, we use static mapping, in which each node configures its own map directly from a stored file at boot time.

It has been shown [10], by modifying the MCP program to disable host DMA transfers, that the network fabric can sustain transfers near the theoretical transfer rates suggested by the network card's clock rate and the data width and that traffic through distinct ports does not interfere, although there can be head-of-line blocking when two packets compete for the same input port. In practice, however, the limitations of the host I/O bus and other overhead associated with the host can reduce the point-to-point bandwidth and increase the network latency.

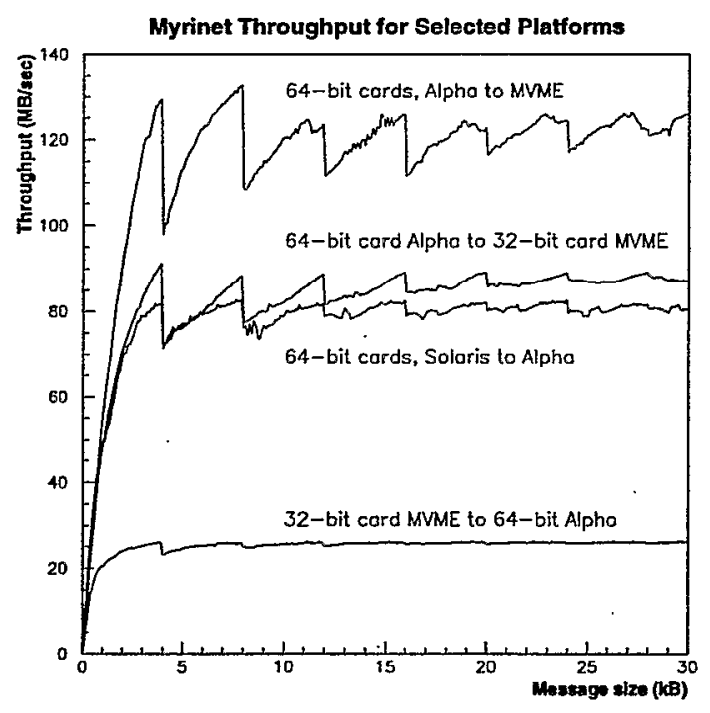

Figure 2: Myrinet throughput as a function of message size for selected platforms.

Several different models of the Myrinet network card are used in the STAR DAQ/L3 system [11]. These cards break into two groups, cards with LANai 4.03 processors and 32bit PCI connections, and cards with LANai 7.02 processors and 64-bit PCI connections. Furthermore, the components of the DAQ system include MVME processors running VxWorks, Compaq Alpha workstations-running Linux, and SUN Ultrasparc workstations running Solaris. We have also 
performed Myrinet tests with Intel Pentium processors running Linux. We found no problems with the interoperability of the network between the various platforms. The Myrinet throughput as a function of message size is shown in Figure 2 for selected platforms. These curves were produced using gm_allsize, a test program distributed with the GM driver. The $130 \mathrm{MB} / \mathrm{sec}$ throughput of the top curve, showing transfers from an Alpha to MVME, is typical performance between two 64-bit cards. The scalloped periodicity of the throughput is due to the $4 \mathrm{~K}$ maximum packet size of the underlying network protocol. If either the sender or the receiver is a 32-bit card, one obtains a maximum throughput of $85 \mathrm{MB} / \mathrm{sec}$ as demonstrated by a 64-bit card Alpha sending to a 32-bit card MVME. The bottom two curves show two important exceptions to the above rules. The first is that the throughput sending from the Ultrasparc Solaris is limited $80 \mathrm{MB} / \mathrm{sec}$ even though both the sender and receiver are using 64-bit cards. It is important to note, however that the full $130 \mathrm{MB} / \mathrm{sec}$ performance is recovered if the Solaris machine is receiving the data. The final curve shows that sending from MVME processors with 32-bit cards has a throughput of only 25 $\mathrm{MB} / \mathrm{sec}$ due to an incompatibility with the DMA burst mode on the MVME PCI bus. This problem is fixed in the newer 64-bit cards, but is relevant to our system because these are the cards currently installed in the DET components. This does not limit the final DAQ throughput, because we are limited by the total bandwidth of the single receiving node, rather than the individual bandwidths of the sending nodes.

\section{IMPLEMENTING MYRINET}

In this section, we first describe the functionality provided by the Myrinet driver, GM. Then we will describe the software architecture of the STAR DAQ system to define our requirements. Finally, we will describe myriLib, our implementation library for Myrinet in the STAR/DAQ.

\section{A. GM Functionality}

The Myrinet driver, GM, provides a set of user calls to send and receive data. There are two supported modes of operation, messages and directed sends. Messages may be of any length, so long as the host programs keep DMA accessible buffers of the appropriate lengths supplied to the GM driver for sending and receiving. When an application receives a message, it must handle the message and then return the buffer to the driver for re-use. Similarly, a user program may not write into the buffer of a sent message until it has received a callback from the GM driver indicating that the message has been transmitted. Messages are read explicitly by the receiver by polling, or blocking on a receive message call. In contrast, directed sends are placed directly into registered host memory of the receiving node via DMA transfer with no notification to the receiver. GM messages include control messages to the driver which must be handled and this imposes structure on the user program centered around the receive message call.

There are several additional considerations regarding the GM driver. Although GM provides independent "ports" to the network interface card, the number of these ports is limited, particularly on the older 32-bit cards and GM calls for the same port are not thread-safe, nor can separate Unix processes access the same port. Secondly, GM does not handle byte swapping of messages between big endian and little endian machines. Finally, the Solaris operating system does not support DMA memory registration for more than $\sim 5 \mathrm{MB}$ chunks. In contrast, for the Buffer Box, and year 2 Event Builder directed sends to data areas as large as $1.5 \mathrm{~GB}$ are required.

\section{B. STAR DAQ Software Architecture}

The bulk of the STAR DAQ software is message based. Typically, each CPU runs multiple tasks so two numbers, the Node ID and Task ID, identify a task. Each node maintains an array of message queues, one for each running task. The L3 trigger tasks run within a single thread, and so the message queues are simple software queues. In Solaris, tasks run in separate processes, and the queue library is based upon named pipes. Each task is essentially a loop which first reads a message from its own queue and then executes code based on the message contents. We use a custom message protocol (ICCP) which specifies 120 byte messages consisting of a standard header and optional payload. Because multiple events are processed at the same time, messages are fully asynchronous. Data transfers are performed using direct DMA to the receiver's memory.

To facilitate platform independent, network independent communications, we have implemented a message sending function daqMsgSend() which is configured to route messages to the appropriate destination via the Myrinet, VME, Ethernet, or a local queue. In the same way, each network has an associated task that receives messages, determines the destination task and places the message in the appropriate queue. In this way, there is no need for explicit read calls to any network. All messages come in through a unified queue.

\section{The myriLib Library}

The primary design criteria for the implementation of Myrinet in the STAR DAQ system was that the transition from the SCI network to the Myrinet network be accomplished as seamlessly as possible. To this end we implemented a library with two main functions. The first, myriMsgSend() sends ICCP messages to remote nodes. The second, myriMemcpy( $)$ has the semantics of the memcpy () function call, but copies data via direct sends to remote nodes. There are several flavors of the myriLib library depending on whether the host node tasks run as threads or processes (we consider VxWorks tasks to be threads for the purpose of this discussion) and whether the host node requires intermediate buffering for large myriMemcpy() transfers.

Figure 3 shows the structure of the threaded version of myrilib. The myriRcv thread processes GM driver commands, handles GM callback functions, and routes incoming messages to the appropriate queue. Calls to the send functions are initiated in the context of the user thread, but the buffer management and GM callback functions are serviced within the context of the myriRcv thread. GM calls between the various threads are protected using semaphores. The . myriCpySend thread is used for processing 


\section{YEAR TWO EVENT BUILDER}

For year 1 running, the Event Builder was implemented on a MVME processor. The events were built in a $256 \mathrm{MB}$ extended PCI based RAM. After an event was built, it was transferred over Myrinet to the Solaris BB node for shipment to the RCF facility for final storage. This architecture was necessary in the original design of the DAQ system because there was no Solaris support for the SCI network cards. The multi-platform success of Myrinet opened the possibility of moving the Event Builder to the Buffer Box node.

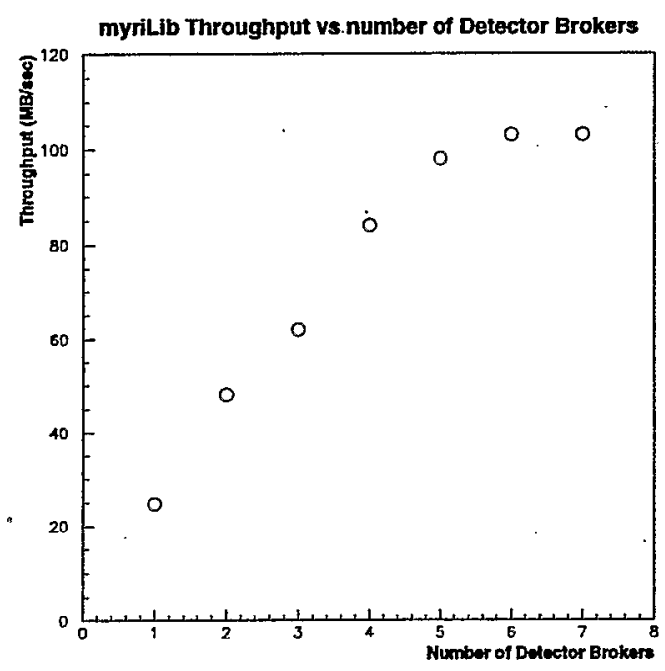

Figure 6: Process myriMemcpy0 throughput to Solaris as a function of number of 32-bit card MVME senders.

There are several major advantages for the new architecture. First, it removes a node from the network representing a significant simplification of the DAQ software and network protocol. It replaces the point-to-point data transfer between the EVB and Solaris BB node with a multiple sender transfer. The performance enhancement is further extended by the observation that simultaneous incoming and outgoing traffic on the extended PCI memory of the year 1 EVB had restricted DAQ throughput to the $B B$ to $-28 \mathrm{MB} / \mathrm{sec}$.

The second advantage of the new architecture is the huge increase in available memory for event building. This corresponds directly to simplifications in the Event Building software and should increase the maintainability of the code. In addition, the current limitation on the total DAQ throughput is $\sim 25 \mathrm{MB} / \mathrm{sec}$. The source of this limitation is the disk performance of the RCF. HPSS system, but we have demonstrated that by opening multiple streams of data to this system we can write to multiple disks increasing the throughput 30-35 MB/sec. The overhead of the transfer mechanism to RCF requires large sends $(-100 \mathrm{MB})$ to obtain high performance, so the extra memory can potentially lead to direct throughput enhancements.

The final advantage of this change is the processing power of the multi-CPU Solaris machine. This opens the possibility of performing compression on the built events to increase the effective throughput of the DAQ system.
Our implementation of the EVB for Solaris builds events in a large (1.5GB) shared memory buffer. The entire buffer is locked into real memory to prevent virtual memory swapping. The memory is managed by the EVB task that sends messages to separate taping processes to store events. The data are sent to the RCF over Gigabit Ethernet using a version of PFTP customized to operate from memory buffers rather than disk. Preliminary timing results with the combined BB/EVB show small event performance of 140 evts/sec, and sustained data rates to the EVB of up to 100 $\mathrm{MB} / \mathrm{sec}$.

\section{DAQ PERFORMANCE AND RELIABILITY}

The year 1 RHIC run lasted for approximately 3 months. During this time, stable beam was available for an integrated time on the order of 15 days. The STAR detector took data for a significant portion of this time, with very little downtime due to the DAQ system. During this time, the STAR DAQ system recorded $\sim 10 \mathrm{~TB}$ of data, consisting of $\sim 2.03$ Million events. When the beam luminosity was sufficient, central events were recorded to tape at the RHIC Computing Facility at rates of $\sim 3$ Events/sec with a peak bandwidth $\sim 25$ $\mathrm{MB} / \mathrm{sec}$. Minimum Bias events were recorded at rates of -15 Events/sec.

The reliability of the Myrinet network was extraordinary. There were 4 known message transmission failures. Although the specific cause of the 4 events was not determined, these each occurred in sends from the same node, and may indicate a fault on the specific network interface card. These faults were each reported to the host software by the Myrinet driver. There has been no data corruption traced to network malfunctions.

\section{CONCLUSIONS •}

In this paper we have described the implementation of a Myrinet event building network for the STAR DAQ system. We measured the Myrinet performance for all hardware and software platforms used in the STAR DAQ system, for several different versions of the Myrinet network cards. The implementation of the network was based upon the standard Myrinet driver, GM. This driver was supplemented by a STAR-specific library myriLib, which provides a convenient interface for DAQ software. The Myrinet network allowed us to move the central Event Builder node to a Solaris workstation for Year 2 operations. This architecture change increased the event building rate of the network to $\sim 100$ $\mathrm{MB} / \mathrm{sec}$, although the total $\mathrm{DAQ}$ throughput is still limited to $\sim 25 \mathrm{MB} / \mathrm{sec}$ by the disk writing speed for the HPSS tape system at RCF. First year performance of the STAR detector demonstrates the suitability and reliability of the Myrinet event building network in the STAR DAQ system.

\section{ACKNOWLEDGMENTS}

This work is supported in part by the U.S. Department of Energy under contract No. DE-ACO2-98CH10886. 
myriMemcpy() calls to buffered nodes and will be discussed later. It is never invoked, except when sending to Solaris.

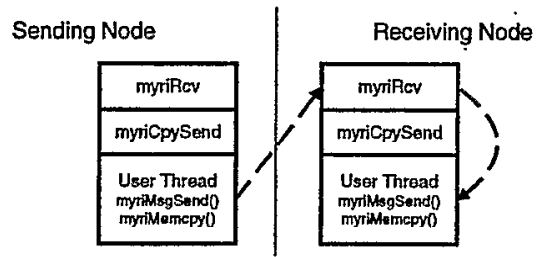

Figure 3: Operation of the thread (VxWorks task) version of myriLib. The myriCpySend task is only used when sending data to Solaris.

The process version of myriLib is very similar to the thread version, but it contains two additional threads, one for messages, and the other for directed sends. These threads initiate the Myrinet send functions on the behalf of user process requests, which are passed via local queues. The data buffers are made visible to both the user process and the myriLib process using shared memory segments.

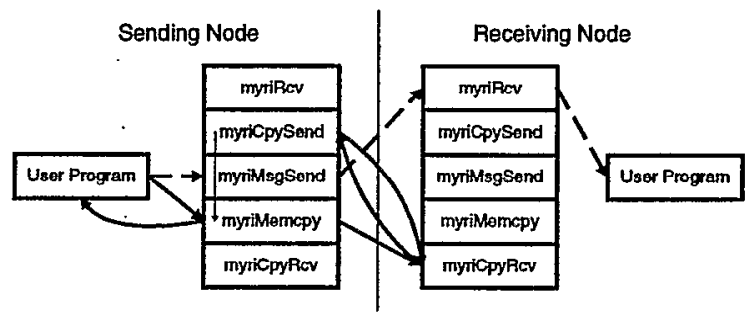

Figure 4: Operation of the process version of myrilib with buffered directed sends. Dashed lines represent the path of a message from the user task of the sender to the user task of the receiver. The solid lines represent the path of control messages sent to manage buffered directed sends.

On Solaris myriMemcpy() function is complicated by the limitation on DMA accessible memory. The requirement of large data sends forces us to break the send buffer into smaller chunks, to send each chunk to the receiving node individually, and then to copy the buffers into the shared memory segment of the receiver as they arrive. The management of these buffers requires two further threads, myriCpySend and myriCpyRcv. The solid lines in Figure 4 illustrate how this works. The user process on the sender node places a message containing the address and size of the memory buffer to be moved, as well as the destination address into the myriMemcpy thread's queue. This thread divides the buffer into manageable chunks and sends a Myrinet message to the destination's myriCpyRcv announcing the first chunk. The receiving myriCpyRcv thread allocates a buffer and passes its address back to the myriCpySend thread on the sending node. This initiates an exchange between the myriCpySend thread of the sender and the myrCpyRcv thread of the receiver. The myriCpySend function performs a directed send of the chunk, and then announces the next chunk. The myriCpyRcv thread copies the buffer to its proper location in shared memory and returns a message with the next buffer. When the transfer is complete, the myriCpySend function lowers a semaphore set by the myriMemcpy thread (vertical arrow in Figure 4) which causes a message to be returned to the user program which then returns from the myriMemcpy() function call.
The introduction of the myriLib layer introduces some overhead from task switching and thread synchronization as compared to the single-thread gm_receive() based test program gm_allsize. The myriLib half round trip latency for messages is $-45 \mu \mathrm{Sec}$ for the 32-bit Myrinet cards, significantly longer than the $20-30 \mu$ Sec latency seen with gm_allsize. In the process version of myriLib on Solaris the latency can be as high as $110+/-30 \mu \mathrm{Sec}$ due to the extra overhead of process context switching.

Figure 5 shows the point to point myriMemcpy() throughput as a function of data size for selected platforms. Note that the horizontal axis is expanded compared to Figure 2. The top line shows that the typical throughput between two 64-bit Myrinet cards using myriLib approaches that of gm_allsize. The bottom line shows the sending performance of the 32-bit cards on MVME processors and is analogous to that of Figure 2.

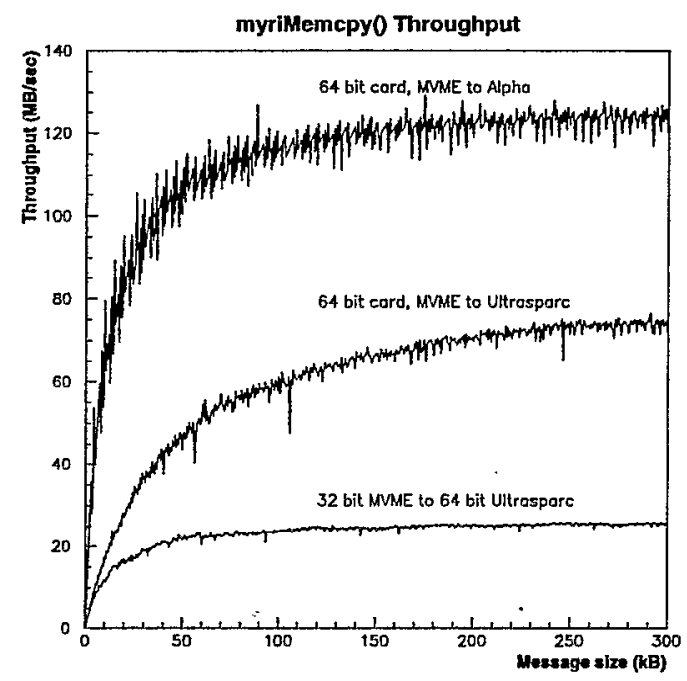

Figure 5: myriMemcpy() throughput as a function of data size for selected platforms.

The center curve in Figure 5 shows the performance of a 64-bit Myrinet card sending to a buffered Solaris node. Although the point to point myriMemcpy 0 throughput is significantly degraded when sending to Solaris by the need to copy the memory from the receiving buffer, this is of little relevance to the performance of the STAR DAQ system because multiple sending nodes rapidly restore the aggregate bandwidth to normal values. This is because the DMA transfer into one buffer can occur while the CPU is copying another node's buffer. Figure 6 shows the myriMemcpy() throughput to Solaris as a function of the number of senders sending from 32-bit cards on MVME processors. The throughput increases linearly until a limiting bandwidth of $100 \mathrm{MB} / \mathrm{sec}$ is reached at six nodes. The $100 \mathrm{MB} / \mathrm{sec}$ bandwidth is reached for two sending nodes for other sending platforms. 